\title{
Literaturhinweise
}

\section{Allgemeine Staatslehre}

\author{
Burkhard Schöbener/Matthias Knauff, 2. Auflage, Verlag C. H. Beck, München 2013, \\ 338 S., $23,90 €$
}

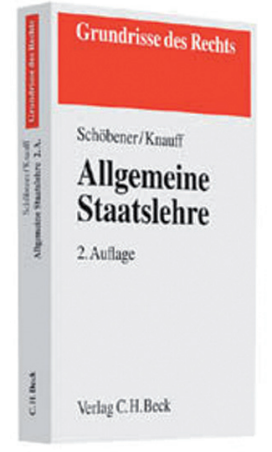

Die Allgemeine Staatslehre wird genauso wie die anderen Grundlagendisziplinen in der juristischen Ausbildung weitgehend vernachlässigt. Prüfungsordnungen und Studienpläne sehen eine Befassung mit ihr in der Regel nicht verpflichtend vor. Dabei ist die Allgemeine Staatslehre Grundvoraussetzung für das Verständnis von Verfassungs- und Völkerrecht. Im Zusammenspiel mit den Grundlagendisziplinen Rechtsgeschichte, Rechtsphilosophie und Rechtsvergleichung geht sie den wesentlichen Fragen des menschlichen Zusammenlebens nach: Was ist Staatlichkeit und wozu dient diese? Was sind die Aufgaben eines Staates? Welche Staats- und Regierungsformen lassen sich unterscheiden? Was versteht man unter Demokratie und Rechtsstaatlichkeit? Viele dieser Fragen werden zumindest ansatzweise im Rahmen der verfassungs- bzw. staatsrechtlichen Studienliteratur angesprochen, aber nicht in der notwendigen Tiefe behandelt. Deshalb ist die Lektüre eines Lehrbuches zur Allgemeinen Staatslehre für denjenigen, der Verfassungs- und Völkerrecht ernsthaft verstehen und begreifen will, unverzichtbar.

In der modernen Studienliteratur scheint sich nun neben dem bisherigen Standardwerk von Reinhold Zippelius mit Erscheinen der 2. Auflage das Lehrbuch von Burkhard Schöbener und Matthias Knauff zu etablieren. Mit einer Fixierung des Untersuchungsgegenstandes, einer Darstellung der Methodik und einer Abgrenzung zu anderen Wissenschaftszweigen leiten die Autoren ihr Lehrbuch ein. Im zweiten und dritten Abschnitt führen sie den Leser über die Erläuterung der historischen Grundlagen an den Begriff des Staates heran. Sodann werden im vierten Abschnitt Rechtfertigung, Funktionen, Ziele und Aufgaben des Staates behandelt. Die staatsphilosophischen Theorien zur Legitimation des Staates werden dabei zwar nicht erschöpfend, aber doch im Wesentlichen umfassend dargestellt. Nach einem Exkurs zu Widerstand und Revolution beschreiben die Autoren die bedeutendsten Modelle zur Einteilung der Staatsformen. Der folgende Teil über die Regierungsformen ist allerdings etwas zu kurz geraten. Eine ausführlichere Darstellung der unterschiedlichen Ausprägungen der demokratischen und monarchischen Regierungssysteme wäre wünschenswert gewesen. So wird zum Beispiel das semipräsidentielle Regierungssystem leider überhaupt nicht erwähnt. Im Anschluss an die Regierungsformenlehre wird im fünften Abschnitt auf die Bedeutung der Grund- und Menschenrechte sowie der verfassungsstaatlichen Grundprinzipien für den modernen Verfassungsstaat eingegangen. Der sechste Abschnitt hat den Einheitsstaat und die Staatenverbindungen zum Gegen- 
stand. Im letzten Abschnitt thematisieren die Autoren die Rolle des Staates in der Völkergemeinschaft bzw. der internationalen Rechtsordnung.

Insgesamt verschafft der vorliegende Band dem Leser in vorwiegend eingängiger Sprache einen guten Überblick über die Allgemeine Staatslehre und kann den Studierenden ohne Einschränkung empfohlen werden. Wer sich tiefergehender mit der Materie beschäftigen möchte, erhält durch die zahlreichen Literaturangaben am Schluss eines jeden Abschnittes weiteren Lesestoff. Dabei wird und sollte man um eine Lektüre der Grundlagenwerke von Platon, Aristoteles, Machiavelli, Locke, Hobbes, Montesquieu oder Rousseau allerdings nicht herumkommen. Schließlich kann kein Lehrbuch zufriedenstellend zusammenfassen und ersetzen, was diese Staatsphilosophen uns hinterlassen haben. 